\title{
Nonlinear parametric oscillations of a viscoelastic shallow shell of variable thickness
}

\author{
Dadakhon Khodzhaev ${ }^{1, *}$, Bakhodir Normuminov ${ }^{1}$, and Yazdan Mustapakulov ${ }^{2}$, and Angela \\ Mottaeva ${ }^{3,4}$ \\ ${ }^{1}$ Tashkent Institute of Irrigation and Agricultural Mechanization Engineers, Kary-Niyazov 39, 100000 \\ Tashkent, Uzbekistan \\ ${ }^{2}$ Tashkent University of Information Technologies, Amir Temur 108, 100200 Tashkent, Uzbekistan \\ ${ }^{3}$ Moscow State University of Civil Engineering, 129337, 26, Yaroslavskoye Shosse, Moscow, Russia \\ ${ }^{4}$ Moscow Region State University, 105005, Radio str, 10A, Moscow, Russia
}

\begin{abstract}
The problem of parametric oscillations of an isotropic viscoelastic shallow shell of variable thickness under periodic load is considered. It is believed that under the influence of specified load, the shallow shell allows displacements (in particular, deflections), commensurate with its thickness. In a geometrically nonlinear statement, taking into account the viscoelastic properties of material, a mathematical model of the problem has been developed using the classical Kirchhoff-Love hypothesis. Using the Bubnov-Galerkin method based on the polynomial approximation of the deflections, the problem is reduced to the study of the system of integro-differential equations, where time is the independent variable. The solution of the system of integrodifferential equations is determined by the proposed numerical method. Based on this method, a numerical solution algorithm is described. The Koltunov-Rzhanitsyn kernel with three different rheological parameters is chosen as a weakly singular kernel. At the same time, the effect of geometric nonlinearity, viscoelastic properties of material, as well as other physicomechanical and geometric parameters and factors (rheological parameters, thickness, initial shape imperfections, aspect ratios, boundary conditions, excitation coefficient) on the area of dynamic instability is taken into account. The results obtained in this study are in good agreement with the results and data obtained by other authors.
\end{abstract}

\section{Introduction}

Modern technology, construction and other spheres of industry employ complex structures, the durability, reliability and high efficiency of which are of great importance. At present, light and durable thin-walled structures such as plates, panels and shells under force loads are widely and effectively used in construction, aircraft and rocket production. To ensure the required rigidity in the right places, thin plates or shells may have smooth swellings. Optimal design of such structures is impossible without creating mathematical models that allow an account of the maximum possible number of factors affecting their performance. In recent years, it has become increasingly important to search for the best configuration, especially to the study of the dynamic problems of thin-walled structures of variable thickness. Therefore, their design scheme should be considered taking into account

\footnotetext{
*Corresponding author: dhodjaev@mail.ru
} 
smooth-variable or step-variable shell thickness. Under significant effects there appear large deflections in such elements; to calculate such structures it is necessary to conduct research in a geometrically nonlinear statement.

In world practice, special attention is paid to the use of light composite materials in construction, this leads to the need to consider the structures with uniform and non-uniform properties of material. The behavior of thin-walled structures has not yet been studied sufficiently due to the difficulty of accounting for the factors listed above and the need to solve cumbersome non-linear boundary value problems.

Therefore, the development of new mathematical models of strain of thin-walled shell structures of variable thickness under various types of loading, as well as effective methods and algorithms for their study is an important task.

A series of publications is devoted to the study of the behavior of plates, panels and shells of constant thickness under dynamic loads in an elastic statement. A review of the results of these studies can be found in [1,2].

Nonlinear dynamics of plates and shallow shells is considered in the studies by A.C. Volmir [2], V.A. Krysko [3] and other authors. The geometrically nonlinear theory of shells of step-variable thickness has been developed by V. Karpov [4].

A review of the study of plates and shells behavior under dynamic loads with regard to the viscoelastic properties of material can be found in [5].

The torques of a rectangular panel with clamped edges as an element of ship structure under uniform load are calculated and analyzed [6], taking into account transverse shear strains; the contribution of the corresponding shear stresses to the overall stress state is considered.

In [7], the shapes of the Kirchhoff square plate with a clamped end are obtained and analyzed before and after the loss of stability in the case of compound bending (uniform transverse load in combination with compressive end load).

Several mathematical models of deformation of reinforced shell structures, including those that account for various properties of the material are described in [8]. For the structures composed of orthotropic and isotropic materials, linearly elastic and physically nonlinear problems, as well as the problems of creep, are considered.

The solution of the plane problems of the theory of elasticity on the basis of the approximation of stresses is considered in [9]. To build the solution, the additional energy functional is used.

Finite element models for plate bending problems are constructed in [10] on the basis of approximations of moment fields. Bending and torsion moments are approximated over the area of finite elements by piecewise constant functions.

Research carried out in [11] gives a solution to the problem of bending of a geometrically nonlinear cantilever rod according to the theories of Kirchhoff and Cosserat-Tymoshenko with the subsequent comparison of the results obtained.

Two models of deformation of reinforced orthotropic shells under dynamic load are considered in [12]. Mathematical models are based on the Kirchhoff-Love hypothesis of shell theory. The models take into account geometrical nonlinearity, properties of an orthotropic material and reinforcement elements.

In [13] nonlinear vibrations of viscoelastic thin rectangular plates subjected to normal harmonic excitation in the spectral neighborhood of the lowest resonances are investigated. The von Karman nonlinear strain-displacement relationships are used and geometric imperfections are taken into account.

In [14], a dynamic impulse bending of a double curvature shell clamped on all edges is investigated using the Novozhilov nonlinear theory of shells and the Lagrange equation of motion. 
The behavior of an elastic shallow shell under external load is studied in [15] by the finite element method for different boundary conditions.

The research in [16] is devoted to the review of the methods for studying linear and nonlinear oscillations of plates and shells.

However, at present, the behavior of plates and shells of smooth-variable thickness, at a joint account of mentioned important factors, is insufficiently studied and requires further study $[17,18]$.

Parametric oscillations have become the subject of numerous studies applied to various mechanical systems with distributed parameters, in particular to rods, plates and shells.

The effect of initial imperfections on the parametric vibrations of cylindrical shells is analyzed in [19]. The shell has moderate amplitudes of vibrations; therefore, geometrically nonlinear theory is used.

In [20] a problem of obtaining natural oscillation frequencies of an isotropic cylindrical shell with variable thickness and density along the generatrix is considered based on V.Z. Vlasov's semi-membrane theory. For one relation between the change in thickness and density we obtained an exact solution for finding the base oscillation frequency of a cylindrical shell with a variable thickness and density along the generatrix.

A vector-matrix method for solving nondecaying systems of nonlinear integrodifferential equations with variable coefficients and with weakly singular kernels of the hereditary theory of viscoelasticity based on the use of quadrature formulas is proposed in [21].

Despite the numerous published papers devoted to parametric oscillations of thin-walled structures, the number of papers devoted to the dynamic stability of thin-walled structures, with account of viscoelastic properties of material, is very limited [22-25].

\section{Materials and Methods}

In this paper, parametric oscillations of a viscoelastic double curvature shell with sides a and $\mathrm{b}$, variable thickness $\mathrm{h}=\mathrm{h}(\mathrm{x}, \mathrm{y})$ and curvature radii of the middle surface $\mathrm{R} 1$ and $\mathrm{R} 2$ under axial dynamic loads (see Fig.1) are investigated. Let the shell be dynamically loaded along the side a with a periodic load $\mathrm{P}(\mathrm{t})=\mathrm{P} 0+\mathrm{P} 1 \cos \Theta \mathrm{t}(\mathrm{P} 0, \mathrm{P} 1=$ const; $\Theta$ is the frequency of the external periodic load).

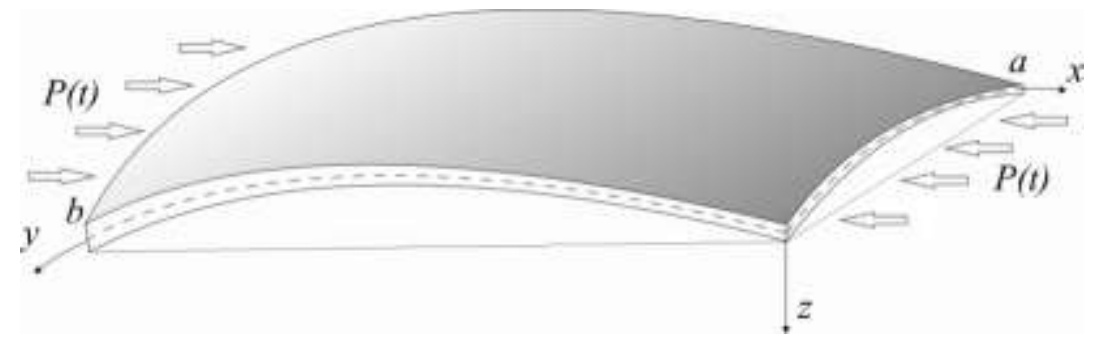

Fig. 1. Shallow shell of double curvature of variable thickness.

Under accepted assumptions, accounting the force $P(t) \frac{\partial^{2} w}{\partial x^{2}}$, the mathematical model of this problem with respect to the deflection $w=w(x, y, t)$ and displacements $u=u(x, y, t), v=v(x, y, t)$ is described by equation [26]

$$
\left(1-\Gamma^{*}\right)\left\{h \left[\frac{\partial^{2} u}{\partial x^{2}}-\left(k_{x}+\mu k_{y}\right) \frac{\partial w}{\partial x}+\frac{1-\mu}{2} \frac{\partial^{2} u}{\partial y^{2}}+\frac{1+\mu}{2} \frac{\partial^{2} v}{\partial x \partial y}+\frac{\partial w}{\partial x} \frac{\partial^{2} w}{\partial x^{2}}+\right.\right.
$$




$$
\begin{aligned}
& \left.+\frac{1-\mu}{2} \frac{\partial w}{\partial x} \frac{\partial^{2} w}{\partial y^{2}}+\frac{1+\mu}{2} \frac{\partial w}{\partial y} \frac{\partial^{2} w}{\partial x \partial y}\right]+\frac{\partial h}{\partial x}\left[\frac{\partial u}{\partial x}+\mu \frac{\partial v}{\partial y}-\left(k_{x}+\mu k_{y}\right) w+\right. \\
& \left.\left.+\frac{1}{2}\left(\frac{\partial w}{\partial x}\right)^{2}+\frac{\mu}{2}\left(\frac{\partial w}{\partial y}\right)^{2}\right]+\frac{1-\mu}{2} \frac{\partial h}{\partial y}\left(\frac{\partial u}{\partial y}+\frac{\partial v}{\partial x}+\frac{\partial w}{\partial x} \frac{\partial w}{\partial y}\right)\right\}+p_{x}-\frac{\left(1-\mu^{2}\right) \mathrm{p} h}{E} \frac{\partial^{2} u}{\partial t^{2}}=0 \\
& \left(1-\Gamma^{*}\right)\left\{h \left[\frac{\partial^{2} v}{\partial y^{2}}-\left(k_{y}+\mu k_{x}\right) \frac{\partial w}{\partial y}+\frac{1-\mu}{2} \frac{\partial^{2} v}{\partial x^{2}}+\frac{1+\mu}{2} \frac{\partial^{2} u}{\partial x \partial y}+\frac{\partial w}{\partial y} \frac{\partial^{2} w}{\partial y^{2}}+\right.\right. \\
& \left.+\frac{1-\mu}{2} \frac{\partial w}{\partial y} \frac{\partial^{2} w}{\partial x^{2}}+\frac{1+\mu}{2} \frac{\partial w}{\partial x} \frac{\partial^{2} w}{\partial x \partial y}\right]+\frac{\partial h}{\partial y}\left[\frac{\partial v}{\partial y}+\mu \frac{\partial u}{\partial x}-\left(k_{y}+\mu k_{x}\right) w+\right. \\
& \left.\left.+\frac{1}{2}\left(\frac{\partial w}{\partial y}\right)^{2}+\frac{\mu}{2}\left(\frac{\partial w}{\partial x}\right)^{2}\right]+\frac{1-\mu}{2} \frac{\partial h}{\partial x}\left(\frac{\partial u}{\partial y}+\frac{\partial v}{\partial x}+\frac{\partial w}{\partial x} \frac{\partial w}{\partial y}\right)\right\}+p_{y}-\frac{\left(1-\mu^{2}\right) \rho h}{E} \frac{\partial^{2} v}{\partial t^{2}}=0 \\
& \left(1-\Gamma^{*}\right) h^{3}\left(\frac{\partial^{4} w}{\partial x^{4}}+2 \frac{\partial^{4} w}{\partial x^{2} \partial y^{2}}+\frac{\partial^{4} w}{\partial y^{4}}\right)+3\left(1-\Gamma^{*}\left[2 h\left(\frac{\partial h}{\partial x}\right)^{2}+h^{2} \frac{\partial^{2} h}{\partial x^{2}}\right]\left(\frac{\partial^{2} w}{\partial x^{2}}+\mu \frac{\partial^{2} w}{\partial y^{2}}\right)+\right. \\
& +6\left(1-\Gamma^{*}\right) h^{2} \frac{\partial h}{\partial x}\left(\frac{\partial^{3} w}{\partial x^{3}}+\frac{\partial^{3} w}{\partial x \partial y^{2}}\right)+6\left(1-\Gamma^{*}\right) h^{2} \frac{\partial h}{\partial y}\left(\frac{\partial^{3} w}{\partial y^{3}}+\frac{\partial^{3} w}{\partial x^{2} \partial y}\right)+ \\
& +3\left(1-\Gamma^{*}\left[2 h\left(\frac{\partial h}{\partial y}\right)^{2}+h^{2} \frac{\partial^{2} h}{\partial y^{2}}\right]\left(\frac{\partial^{2} w}{\partial y^{2}}+\mu \frac{\partial^{2} w}{\partial x^{2}}\right)+\right. \\
& +6(1-\mu)\left(1-\Gamma^{*}\right)\left[2 h \frac{\partial h}{\partial x} \frac{\partial h}{\partial y}+h^{2} \frac{\partial^{2} h}{\partial x \partial y}\right] \frac{\partial^{2} w}{\partial x \partial y}- \\
& -12\left(1-\Gamma^{*}\right) h\left[\left(k_{x}+\mu k_{y}\right) \frac{\partial u}{\partial x}+\left(\mu k_{x}+k_{y}\right) \frac{\partial v}{\partial y}-\left(k_{x}^{2}+k_{y}^{2}+2 \mu k_{x} k_{y}\right) w+\right. \\
& \left.+\frac{k_{x}+\mu k_{y}}{2}\left(\frac{\partial w}{\partial x}\right)^{2}+\frac{k_{y}+\mu k_{x}}{2}\left(\frac{\partial w}{\partial y}\right)^{2}\right]- \\
& -12 \frac{\partial w}{\partial x}\left(1-\Gamma^{*}\right)\left\{h \left[\frac{\partial^{2} u}{\partial x^{2}}-\left(k_{x}+\mu k_{y}\right) \frac{\partial w}{\partial x}+\right.\right. \\
& \left.+\frac{1-\mu}{2} \frac{\partial^{2} u}{\partial y^{2}}+\frac{1+\mu}{2} \frac{\partial^{2} v}{\partial x \partial y}+\frac{\partial w}{\partial x} \frac{\partial^{2} w}{\partial x^{2}}+\frac{1-\mu}{2} \frac{\partial w}{\partial x} \frac{\partial^{2} w}{\partial y^{2}}+\frac{1+\mu}{2} \frac{\partial w}{\partial y} \frac{\partial^{2} w}{\partial x \partial y}\right]+\frac{\partial h}{\partial x}\left[\frac{\partial u}{\partial x}+\right.
\end{aligned}
$$




$$
\begin{gathered}
\left.\left.+\mu \frac{\partial v}{\partial y}-\left(k_{x}+\mu k_{y}\right) w+\frac{1}{2}\left(\frac{\partial w}{\partial x}\right)^{2}+\frac{\mu}{2}\left(\frac{\partial w}{\partial y}\right)^{2}\right]+\frac{1-\mu}{2} \frac{\partial h}{\partial y}\left(\frac{\partial u}{\partial y}+\frac{\partial v}{\partial x}+\frac{\partial w}{\partial x} \frac{\partial w}{\partial y}\right)\right\}- \\
-12 \frac{\partial^{2} w}{\partial x^{2}}\left(1-\Gamma^{*}\right) h\left[\frac{\partial u}{\partial y}+\mu \frac{\partial v}{\partial y}-\left(k_{x}+\mu k_{y}\right) w+\frac{1}{2}\left(\frac{\partial w}{\partial x}\right)^{2}+\frac{\mu}{2}\left(\frac{\partial w}{\partial y}\right)^{2}\right]- \\
-12 \frac{\partial w}{\partial y}\left(1-\Gamma^{*}\right)\left\{h \left[\frac{\partial^{2} v}{\partial y^{2}}-\left(k_{y}+\mu k_{x}\right) \frac{\partial w}{\partial y}+\frac{1-\mu}{2} \frac{\partial^{2} v}{\partial x^{2}}+\frac{1+\mu}{2} \frac{\partial^{2} u}{\partial x \partial y}+\right.\right. \\
\left.+\frac{\partial w}{\partial y} \frac{\partial^{2} w}{\partial y^{2}}+\frac{1-\mu}{2} \frac{\partial w}{\partial y} \frac{\partial^{2} w}{\partial x^{2}}+\frac{1+\mu}{2} \frac{\partial w}{\partial x} \frac{\partial^{2} w}{\partial x \partial y}\right]+\frac{\partial h}{\partial y}\left[\frac{\partial v}{\partial y}+\mu \frac{\partial u}{\partial x}-\right. \\
\left.\left.-\left(k_{y}+\mu k_{x}\right) w+\frac{1}{2}\left(\frac{\partial w}{\partial y}\right)^{2}+\frac{\mu}{2}\left(\frac{\partial w}{\partial x}\right)^{2}\right]+\frac{1-\mu}{2} \frac{\partial h}{\partial x}\left(\frac{\partial u}{\partial y}+\frac{\partial v}{\partial x}+\frac{\partial w}{\partial x} \frac{\partial w}{\partial y}\right)\right\}- \\
-12 \frac{\partial^{2} w}{\partial y^{2}}\left(1-\Gamma^{*}\right) h\left[\frac{\partial v}{\partial y}+\mu \frac{\partial u}{\partial y}-\left(k_{y}+\mu k_{x}\right) w+\frac{1}{2}\left(\frac{\partial w}{\partial y}\right)^{2}+\frac{\mu}{2}\left(\frac{\partial w}{\partial x}\right)^{2}\right]- \\
-12(1-\mu) \frac{\partial^{2} w}{\partial x \partial y}\left(1-\Gamma^{*}\right) h\left(\frac{\partial u}{\partial y}+\frac{\partial v}{\partial x}+\frac{\partial w}{\partial x} \frac{\partial w}{\partial y}\right)+\frac{12\left(1-\mu^{2}\right) \rho h}{E} \frac{\partial^{2} w}{\partial t^{2}}- \\
-
\end{gathered}
$$

where $k_{x}=1 / R_{1}, k_{y}=1 / R_{2}$.

Assume that the shell has initial deflections $w_{0}=w_{0}(x, y)$.

In a general case, the law of change in shell thickness can be of any type. Consider some of the laws of change in thickness. Let the thickness of a thin-walled structural element vary according to the following law $h(x)=\frac{1}{2} h_{0}[1+\varepsilon \sin (2 r-1) \pi x]$. Figure 2 shows the thickness change profiles for different values of the thickness change parameters.

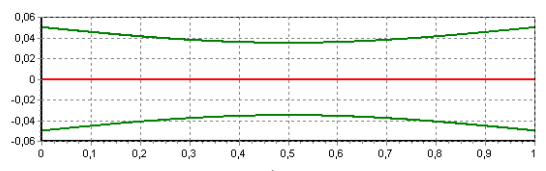

a)

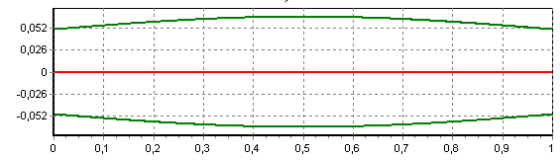

c)

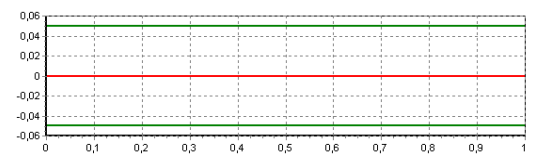

b)

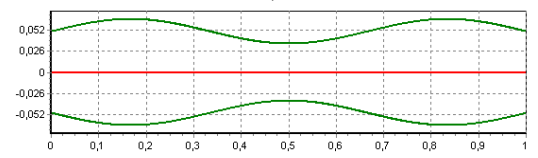

d)

Fig. 2. Change in shell thickness depending on the value of the parameter $r$ : a) $r=0$; b) $r=0.5$; c) $r=1$; d) $r=2$. 
Now consider the case when the thickness varies linearly $h(x)=\frac{1}{2} h_{0}(1+\alpha * x)$. Here, $h_{0}=h(0)=$ const,$\alpha^{*}-$ is the parameter characterizing the thickness variability. Note that this law leads to a linear increase in thickness of structural element in the direction of the $O x$ axis (see Fig.2).

Full and initial deflections, as well as the displacement of the shell are approximated in the form

$$
\begin{aligned}
& u(x, y, t)=\sum_{n=1}^{N} \sum_{m=1}^{M} u_{n m}(t) \phi_{n m}(x, y), v(x, y, t)=\sum_{n=1}^{N} \sum_{m=1}^{M} v_{n m}(t) \varphi_{n m}(x, y), \\
& w(x, y, t)=\sum_{n=1}^{N} \sum_{m=1}^{M} w_{n m}(t) \psi_{n m}(x, y), w_{0}(x, y)=\sum_{n=1}^{N} \sum_{m=1}^{M} w_{0 n m} \psi_{n m}(x, y),
\end{aligned}
$$

where $u_{n m}=u_{n m}(t), v_{n m}=v_{n m}(t), w_{n m}=w_{n m}(t)$ - are unknown time functions; $\phi_{n m}(x, y)$, $\varphi_{n m}(x, y), \quad \psi_{n m}(x, y), \quad n=1,2, \ldots, N ; \quad m=1,2, \ldots, M$ - coordinate functions satisfying the given boundary conditions of the problem.

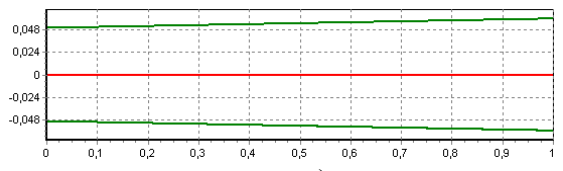

a)

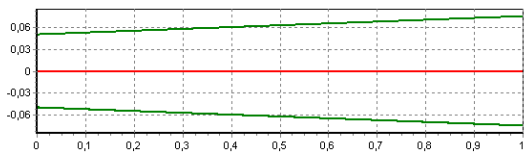

b)

Fig. 3. Change in shell thickness depending on the parameter value $\alpha^{*}:$ a) $\alpha^{*}=0.2$; b) $\alpha^{*}=0.5$.

Substituting (2) into the system of equations (1) and performing the Bubnov-Galerkin procedure, introducing the following dimensionless quantities

$$
\begin{aligned}
& \frac{u}{h_{0}}, \frac{v}{h_{0}}, \frac{w}{h_{0}}, \frac{w_{0}}{h_{0}}, \frac{x}{a}, \frac{y}{b}, \frac{h}{h_{0}}, \lambda=\frac{a}{b}, \delta=\frac{b}{h_{0}}, \bar{k}_{x}=\frac{a^{2}}{h_{0} R_{1}}, \bar{k}_{y}=\frac{b^{2}}{h_{0} R_{2}}, \\
& \bar{q}=\frac{q}{E}\left(\frac{b}{h_{0}}\right)^{4}, \bar{p}_{x}=\frac{p_{x}}{E}, \bar{p}_{y}=\frac{p_{y}}{E}, \frac{\Theta}{\omega}, \omega t, \frac{\Gamma(t)}{\omega}, \delta_{0}=\frac{P_{0}}{P_{c r}}, \delta_{1}=\frac{P_{1}}{P_{c r}},
\end{aligned}
$$

and maintaining the same notation, to determine the unknowns $w_{n m}=w_{n m}(t), u_{n m}=u_{n m}(t)$, $v_{n m}=v_{n m}(t)$, we obtain the following system of nonlinear integro-differential equations:

$$
\begin{gathered}
\sum_{n=1 m=1}^{N} \sum_{k l n m}^{M} a_{n m}-\eta_{1}\left(1-\Gamma^{*}\right)\left\{\sum_{n=1}^{N} \sum_{m=1}^{M}\left(d_{1 k l n m} u_{n m}+\lambda e_{1 k l n m} v_{n m}\right)-\right. \\
\left.\left.-\frac{k_{x}+\lambda^{2} \mu k_{y}}{\lambda \delta} f_{1 k l n m}\left(w_{n m}-w_{0 n m}\right)\right)+\frac{1}{\delta} \sum_{n, i=1}^{N} \sum_{m, j=1}^{M} g_{1 k l n m i j}\left(w_{n m} w_{i j}-w_{0 n m} w_{0 i j}\right)\right\}=0, \\
\sum_{n=1}^{N} \sum_{m=1}^{M} b_{k l n m} \ddot{v}_{n m}-\eta_{2}\left(1-\Gamma^{*}\right)\left\{\sum_{n=1}^{N} \sum_{m=1}^{M}\left(\frac{1}{\lambda} d_{2 k l n m} u_{n m}+e_{2 k l n m} v_{n m}\right)-\right.
\end{gathered}
$$




$$
\begin{gathered}
\left.\left.-\frac{\lambda^{2} k_{y}+\mu k_{x}}{\lambda^{2} \delta} f_{2 k l n m}\left(w_{n m}-w_{0 n m}\right)\right)+\frac{1}{\delta} \sum_{n, i=1}^{N} \sum_{m, j=1}^{M} g_{2 k l n m i j}\left(w_{n m} w_{i j}-w_{0 n m} w_{0 i j}\right)\right\}=0 \\
\sum_{n=1}^{N} \sum_{m=1}^{M} c_{k l n m} \ddot{w}_{n m}+\eta_{3} \sum_{n=1}^{N} \sum_{m=1}^{M} p_{k l n m}^{2}\left(1-2 \mu_{k l n m} \cos \Theta t\right) w_{n m}+ \\
+\eta_{3}\left(1-\Gamma^{*}\right)\left\{\sum_{n=1}^{N} \sum_{m=1}^{M}\left(d_{3 k l n m} u_{n m}+e_{3 k l n m} v_{n m}+f_{3 k l n m}\left(w_{n m}-w_{0 n m}\right)\right)-\right. \\
\left.-\sum_{n, i=1}^{N} \sum_{m, j=1}^{M} g_{3 k l n m i j}\left(w_{n m} w_{i j}-w_{0 n m} w_{0 i j}\right)\right\}- \\
-\eta_{3}\left\{\sum_{n, i=1}^{N} \sum_{m, j=1}^{M} w_{n m}\left(1-\Gamma^{*}\right)\left(d_{4 k l n m i j} u_{i j}+e_{4 k l n m i j} v_{i j}+f_{4 k l n m i j}\left(w_{i j}-w_{0 i j}\right)\right)+\right. \\
\left.+\sum_{n, i, r=1 m, j, s=1}^{N} g_{k l n m i j r s} w_{n m}\left(1-\Gamma^{*}\right)\left(w_{i j} w_{r s}-w_{0 i j} w_{0 r s}\right)\right\}=12 \eta_{3}\left(1-\mu^{2}\right) \lambda^{4} q_{k l} \\
u_{n m}(0)=u_{0 n m}, \dot{u}_{n m}(0)=\dot{u}_{0 n m}, v_{n m}(0)=v_{0 n m}, \dot{v}_{n m}(0)=\dot{v}_{0 n m} \\
w_{n m}(0)=w_{0 n m}, \dot{w}_{n m}(0)=\dot{w}_{0 n m},
\end{gathered}
$$

where $P_{c r}=\frac{\pi^{2}}{3\left(1-\mu^{2}\right)} E\left(\frac{h_{0}}{b}\right)^{2}-$ is the static critical load; $\omega=\sqrt{\pi^{2} E h_{0}^{2} P_{c r}^{*} /\left(\rho b^{4}\right)}-$ is the frequency of major tone of oscillations; $p_{k l n m}^{2}=f_{k l n m}-4 \pi^{2} \lambda^{2} p_{k l n m}^{*} \delta_{0}$; $\mu_{k l n m}=\frac{2 \pi^{2} \lambda^{2} p_{k l n m}^{*}}{p_{k l n m}^{2}} \delta_{1}$; the remaining constant coefficients in this system are related to coordinate functions and their derivatives.

The system of integro-differential equations (3) with the corresponding boundary and initial conditions describes a mathematical model of the problem of nonlinear parametric oscillations of viscoelastic shallow shells of double curvature of variable thickness. Thus, the Mathieu-Hill equation (the third equation of system (3)) corresponding to the nonlinear system under consideration is obtained.

The integration of system (3) is performed using the numerical method proposed in [21]. The weakly singular Koltunov-Rzhanitsyn kernel of the form [27] is used as a relaxation kernel.

\section{Results and discussion}

The results of calculations performed on the computer are reflected in the graphs shown in Figures 4-7.

Figure 4 shows the effect of viscoelastic properties of shell material on its behavior. An analysis of the results obtained shows that an account of viscoelastic properties of material 
leads to a narrowing of the area of dynamic instability.

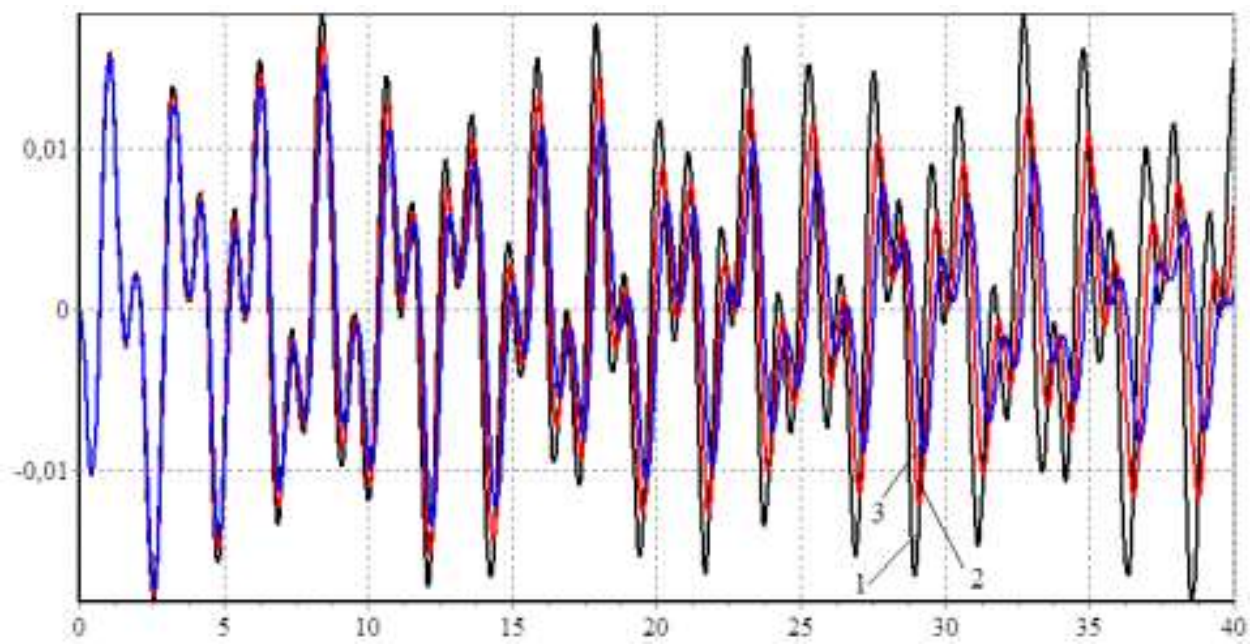

Fig. 4. Dependence of the deflection on time at $\mathrm{A}=0$ (1); 0.05 (2); 0.1 (3).

Figure 5 shows the results of studying shell behavior at different values of the curvature $k_{x}$. The figure shows that an increase in this parameter leads to an increase in the amplitude of oscillations and a phase shifting.

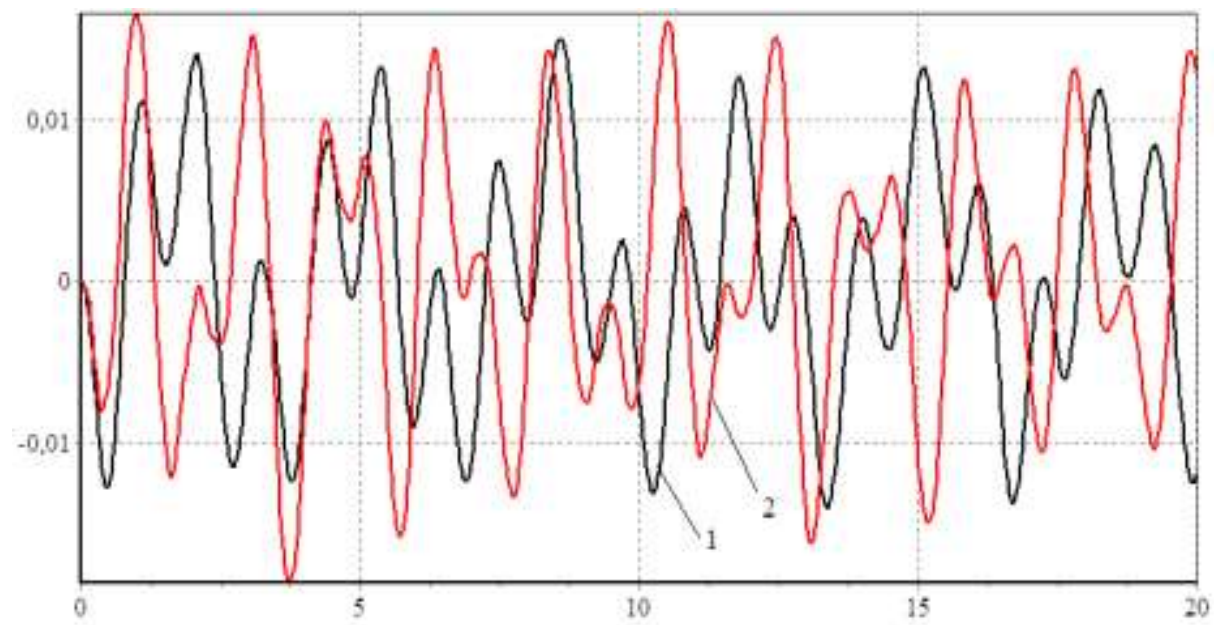

Fig. 5. Dependence of the deflection on time at $k_{x}=0(1) ; 20$ (2).

The results of studying shell behavior at different values of the thickness variation parameter $\alpha^{*}$ are shown in Fig.6. The change in thickness of viscoelastic shell according to the above law, at equal volumes of the shell of constant and variable thickness, leads to a decrease in the maximum displacements. The results of the study show that an increase in this parameter leads to an increase in the amplitude of oscillations and a phase shifting. 


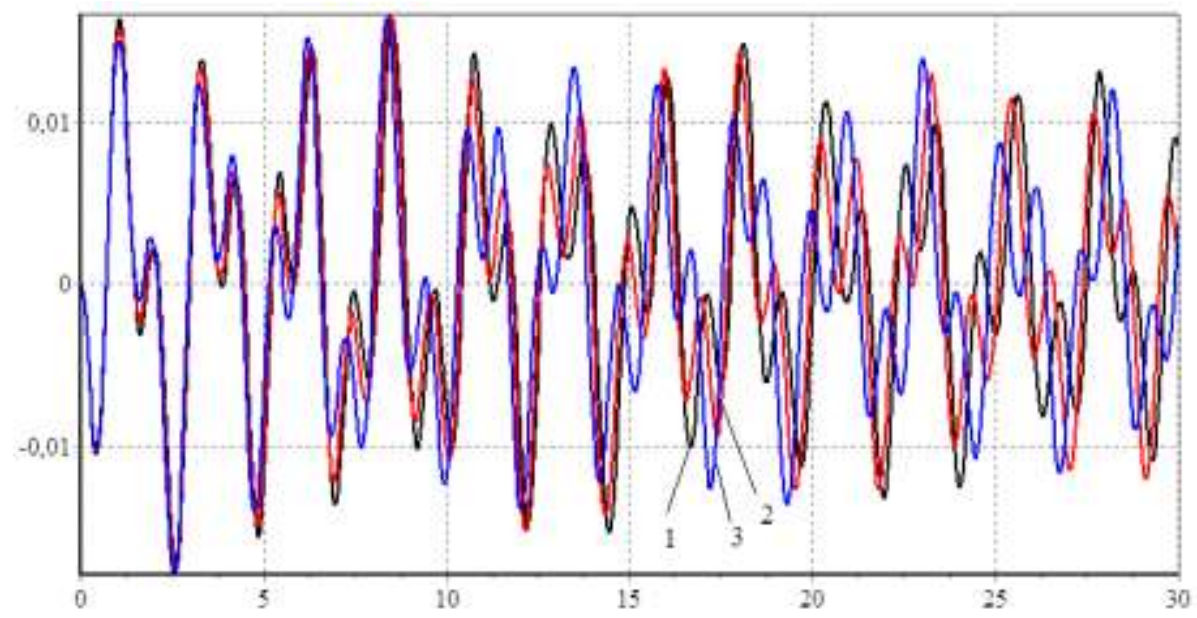

Fig. 6. Dependence of the deflection on time at $\alpha^{*}=0.3$ (1); 0.5 (2); 0.8 (3).

Figure 7 shows the results of studying shell behavior at various values of $\lambda$. The figure shows that an increase in this parameter leads to a decrease in the frequency of oscillations. Numerical results are obtained for the law of thickness variation, shown in Fig.2.

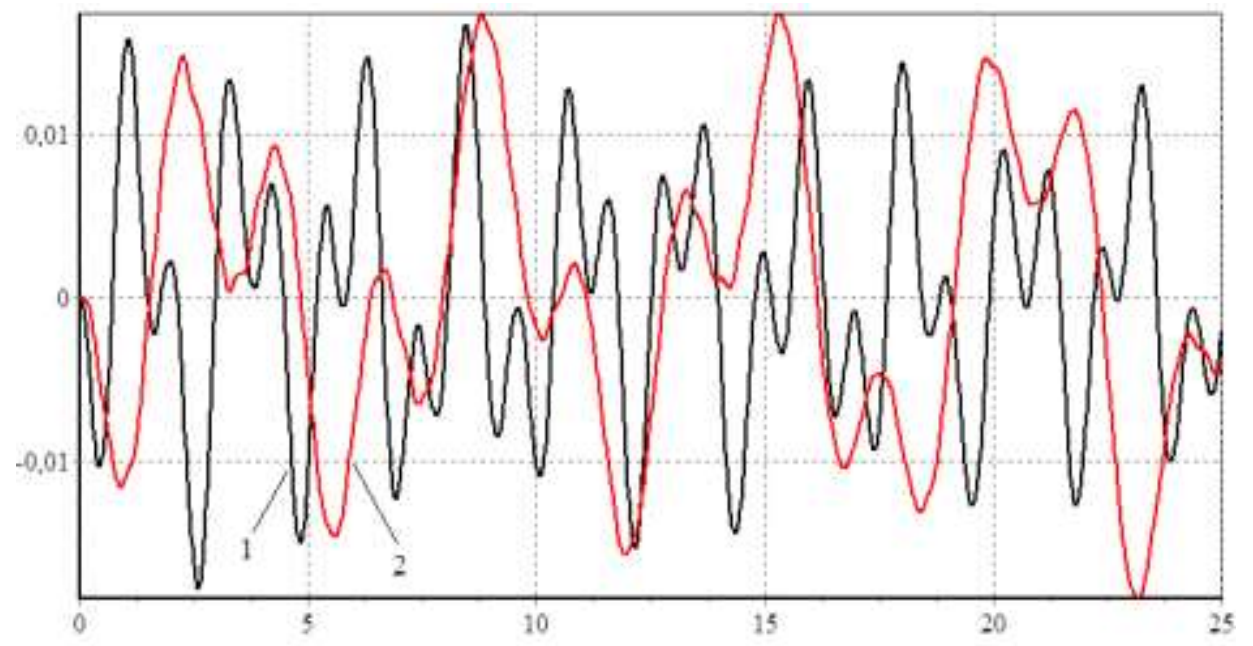

Fig. 7. Dependence of the deflection on time at $\lambda=1(1) ; 2$ (2).

\section{Conclusions}

A mathematical model has been constructed and, in a general form, a system of nonlinear integro-differential equations in displacements with variable coefficients has been obtained for the problems of parametric oscillations of viscoelastic isotropic shallow shells of variable thickness with account of large strains. Resolving systems of nonlinear integro-differential equations of the problem of parametric oscillations of viscoelastic isotropic shallow shells of variable thickness under periodic loads with allowance for large strains have been obtained.

The Bubnov-Galerkin method is used to discretize spatial variables and obtain nondecaying systems of integro-differential equations for the time function of the problems on nonlinear parametric oscillations of viscoelastic shallow shells of variable thickness that 
present the Mathieu-Hill equation in a general form.

An algorithm has been developed for the numerical solution of nondecaying systems of nonlinear integro-differential equations of the problems of nonlinear parametric oscillations of viscoelastic shallow shells of variable thickness, and a set of applied programs has been created based on the developed algorithms, which allows to study the effects of viscoelastic properties of material, rheological parameters, the law of thickness variation, coefficient of excitations and geometric nonlinearities on the dynamic instability areas. As an example, the results of the numerical solution of the problem of nonlinear parametric oscillation of shallow shell of double curvature with hinged-supported edges and linearly variable thickness are presented.

\section{References}

1. V.V. Bolotin, The dynamic stability of elastic systems (Holden-Day, San Francisco, 1964)

2. A.S. Volmir, Stability of deformable systems (Nauka, Moscow, 1967)

3. V.A. Krysko, Nonlinear statics and dynamics of inhomogeneous shells (Publishing House of Saratov University, Saratov, 1976)

4. V.V. Karpov, Geometrically nonlinear problems for plates and shells and methods for solving them (Publishing house ASV, SPbSACU, SPb, 1999)

5. Y.N. Rabotnov, Elements of hereditary solid mechanics (Mir Publishers, Moscow, 1980)

6. M. Sukhoterin, S. Baryshnikov, E. Rasputina, N. Pizhurina, MATEC Web of Conferences (2018)

7. M. Sukhoterin, S. Baryshnikov, T. Knysh, N. Pizhurina, MATEC Web of Conferences (2018)

8. V.V. Karpov, A.A. Semenov, J. Appl. Ind. Math. 11, 70-81 (2017). doi:10.1134/S1990478917010082

9. Y.Y. Tyukalov, Mag. Civ. Eng. 77, 23-37 (2018). doi:10.18720/MCE.77.3

10. Y.Y. Tyukalov, IOP Conf. Series: Materials Science and Engineering (2018)

11. V.V. Lalin, M.O. Belyaev, Mag. Civ. Eng. 53, 39-55+105-16 (2015) doi:10.5862/MCE.53.5

12. A.A. Semenov, J. Sib. Fed. Univ. 9, 485-497 (2016). doi:10.17516/1997-1397-2016-94-485-497

13. M. Amabili, J. Sound Vib. 362, 142-156 (2016). doi:10.1016/j.jsv.2015.09.035

14. D. Sirivolu, M.S. Hoo Fatt, Int. J. Non. Linear. Mech. 77, 281-290 (2015). doi:10.1016/j.ijnonlinmec.2015.09.005

15. J. Harvan, M. Psorty, Procedia Eng. 190, 148-153 (2017). doi:10.1016/j.proeng.2017.05.320

16. M.M. Banerjee, J. Mazumdar, Procedia Eng. 144, 493-503 (2016). doi:10.1016/j.proeng.2016.05.160

17. M.V. Sukhoterin, S.O. Baryshnikov, T.P. Knysh, R.A. Abdikarimov, Mag. Civ. Eng. 82, 81-94 (2018). doi:10.18720/MCE.82.8

18. D. Khodzhaev, R. Abdikarimov, N. Vatin, MATEC Web of Conferences (2018)

19. R. Kochurov, K.V. Avramov, Int. J. Solids Struct. 49, 537-545 (2012). doi:10.1016/j.ijsolstr.2011.10.023

20. A.A. Mochalin, J. Mach. Manuf. Reliab. 44, 434-438 (2015). 
doi:10.3103/S1052618815030127

21. R.A. Abdikarimov, D.A. Khodzhaev, Mag. Civ. Eng. 49, 83-94 (2014). doi:10.5862/MCE.49.9

22. M.M. Mirsaidov, R.A. Abdikarimov, N.I. Vatin, V.M. Zhgutov, D.A. Khodzhaev, B.A. Normuminov, Mag. Civ. Eng. 82, 112-126 (2018). doi:10.18720/MCE.82.11

23. R. Abdikarimov, D. Khodzhaev, N. Vatin, MATEC Web of Conferences (2018)

24. M.M. Mirsaidov, R.A. Abdikarimov, D.A. Khodzhaev, B.A. Normuminov, Vestn. MGSU 13, 1315-1325 (2018) doi:10.22227/1997-0935.2018.11.1315-1325

25. M.M. Mirsaidov, T.Z. Sultanov, R.A. Abdikarimov, A.N. Ishmatov, B.S. Yuldoshev, E.S. Toshmatov, D.P. Jurayev, Mag. Civ. Eng. 77, 101-111 (2018) doi:10.18720/MCE.77.9

26. R.A. Abdikarimov, V.M. Zhgutov, Mag. Civ. Eng. 16, 38-47 (2010)

27. M.A. Koltunov, Creep and relaxation (Vysshaya Shkola, Moscow, 1976) 